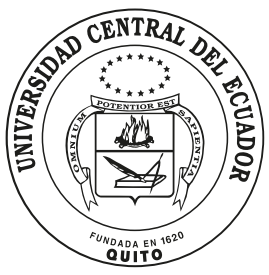

ISSN-i 1390-7042 | ISSN-e 2602-8484

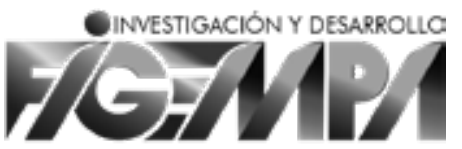

\title{
HUELLA QUÍMICA DE ELEMENTOS IDENTIFICADORES EN LOS DISTRITOS MINEROS NAMBIJA Y ZARUMA-PORTOVELO
}

\section{Chemical footprint of identifying elements in the Nambija and Zaruma-Portovelo mining districts}

Abdón Arturo Enríquez Almeida abdon.enriquez@geoenergia.gob.ec

Instituto de Investigación Geológico y Energético (IIGE)

ORCID: https://orcid.org/0000-0002-7492-1625

\author{
Manuel de Jesús Quezada Ochoa \\ manuel.quezada@geoenergia.gob.ec \\ Instituto de Investigación Geológico y Energético (IIGE) \\ ORCID: https://orcid.org/0000-0003-0421-3006
}

Recibido: marzo de 2020

Aprobado: mayo de 2021

ARTÍ́CULO ORIGINAL

DoI: $10.29166 /$ revfig.v11i1.2181

PALABRAS Clave Concentración, elemento, indicador, muestreo.

KEYWORDS Concentration, element, indicator, sampling.

\section{RESUMEN}

La procedencia del mineral el momento que llega al procesamiento es incierta, razón por la cual se busca metodologías que ayuden a identificar el lugar de extracción y, por ende, la legalidad del mismo. Para efectuar este estudio se tomaron diferentes muestras para efectuar análisis de laboratorio. Se cogieron 150 muestras durante la ejecución del proyecto Rehabilitación Nambija y 78 muestras del proyecto Trazabilidad del oro, desde los frentes de trabajo hasta las plantas de beneficio en Zaruma-Portovelo; ambos proyectos desarrollados por parte del Instituto Nacional de Investigación Geológico Minero Metalúrgico (INIGEMM). Con los resultados de 50 elementos se realizó una interpretación, verificando que hay una diferencia en la concentración de algunos elementos en cada distrito minero, esto se podría usar como indicadores de su procedencia. Así, por ejemplo, para Zaruma-Portovelo tenemos como indicadores Ag y $\mathrm{Pb}$ y para Nambija un grupo de tierras raras (Ce, Er, Gd, Lu, Nd, Y), además de otros elementos como el U, P y Zr.

\begin{abstract}
The origin of the ore is uncertain when to get to the processing, for that reason, this study is looking for methodologies that help to identify the place of extraction and therefore the legality of it. So we took the results of 150 samples from the Nambija Rehabilitation Project and 78 samples of project Gold Traceability from The Work Fronts to the Profit Plants in Zaruma-Portovelo, by National Institute of Geological, Mining \& Metallurgical Research (Instituto Nacional de Investigación Geológico Minero Metalúrgico, INIGEMM), which were analyzed in its lab. With the results of 50 elements an interpretation was made, verifying that there is a difference in concentration of some elements in each mining district, what could be used as indicators of its provenance is so for Zaruma-Portovelo it has $\mathrm{Ag}$ and $\mathrm{Pb}$ indicators and for Nambija a group of rare earths (Ce, Er, Gd, Lu, Nd, Y) in addition to other elements such as U, P and Zr.
\end{abstract}




\section{INTRODUCCIÓN}

Para identificar la procedencia del oro, se optó por buscar elementos químicos que sean representativos en la mineralización de los yacimientos. Con este antecedente se consideraron los datos obtenidos en los proyectos Rehabilitación Nambija y Trazabilidad del oro en frentes de trabajo y plantas de beneficio de Zaruma-Portovelo, donde se obtuvieron 150 y 78 muestras tomadas en diferentes estructuras mineralizadas. Las muestras fueron enviadas a laboratorios químicos, donde se realizaron análisis de varios elementos en común, con los resultados se efectuó un análisis estadístico con el fin de determinar la tendencia de concentración que poseen elementos en particular para cada depósito minero. En el análisis no se consideró al oro, ya que éste es un mineral económicamente rentable para los mineros en los dos distritos mineros.

\section{METODOLOGÍA}

El presente estudio realizó un diagnóstico de la información encontrada en los proyectos Rehabilitación Nambija y Trazabilidad del oro desde los frentes de trabajo hasta las plantas de beneficio. Con estos insumos se pretendía encontrar diferencias entre los dos depósitos mineros. La ubicación de estas dos zonas mineras es en el sur del país. Nambija se encuentra en la provincia de Zamora Chinchipe, Zaruma-Portovelo en la provincia de El Oro. Además, se debe mencionar que ambas zonas tienen diferentes génesis de formación de la mineralización.

\section{GEOLOGÍA}

Geológicamente, el distrito minero Zaruma-Portovelo se encuentra sobre el complejo metamórfico El Oro, la zona de estudio está conformada por tobas, andesitas $\mathrm{y}$ en algunos casos dacitas.

Pratt et al. (1997) indican que la mayor parte del área relacionada con la zona de interés se encuentra sobreyacida por la unidad Portovelo. La serie que conforma la referida unidad geológica está compuesta por rocas de origen volcánico, como basalto y andesita, con cristales predominantes de plagioclasa, anfíbol y augita; así como en menor porcentaje por tobas andesíticas muy meteorizadas y tobas dacíticas. En cambio, Bonilla (2009) indica que la mineralización presente en el distrito corresponde a un epitermal de sulfuración intermedia con presencia de $\mathrm{Au}-\mathrm{Ag} \pm(\mathrm{Cu}, \mathrm{Pb}, \mathrm{Zn})$ pertenecientes a los metales base, así tenemos la presencia de sulfuros como pirita, calcopirita, galena, esfalerita. Además de otros minerales como carbonatos, silicatos (cuarzo, adularia, entre otros).
Por otra parte, en el distrito minero Nambija afloran rocas volcano-sedimentarias de edad jurásica, de color verdoso, con presencia de alteraciones hidrotermales (silicificación, carbonatación, epidotización y presencia de granates), las que se encuentran relacionadas con el skarn, sobreyaciendo a éstas se encuentran rocas volcano-sedimentarias con alteraciones de caolín y arcilla (Litherland et al., 1994).

En el distrito mencionado podemos encontrar granodioritas, diques pequeños de tonalitas-dioríticas, y muy puntualmente lavas andesíticas y basálticas. El mineral de interés en Nambija es el oro, pero se encuentran asociaciones porfídicas de $\mathrm{Cu}-\mathrm{Au}$ y $\mathrm{Cu}-\mathrm{Mo}$. El oro se lo encuentra libre de forma diseminada y con purezas superiores a $90 \%$ dentro de la zona del skarn. Este elemento se lo halla relacionado con vetillas o bolsonadas de cuarzo y menos frecuente con sulfuros, entre los que tenemos pirita y en menor proporción calcopirita, pirrotina, esfalerita y galena. Minerales de hierro como magnetita y hematita

Como se ha señalado anteriormente, las rocas tipo skarn se han formado en diferentes fases, que han afectado de manera variable a los horizontes estratigráficos que se encuentran preservados.

\section{MUESTREO}

Los muestreos en los dos casos se realizaron por medio de canal chip (chip sampling), para lo cual se realizó un corte de 2 " de ancho y 1 " de profundidad perpendicularmente a la estructura mineralizada, la roca extraída fue colocada en plásticos limpios con el fin de realizar el cuarteo y poder extraer muestras con un peso de alrededor de $1 \mathrm{~kg}$.

Del distrito minero Nambija, en los bloques pertenecientes a la concesión minera Asonambile, se extrajeron 150 muestras en el skarn; en cambio en Zaruma-Portovelo se extrajeron 78 muestras de las vetas ubicadas en las concesiones: Bomba de Pachapamba, San Antonio, Palacios, Jorupe, Tres Reyes, Miranda, entre otras.

Posteriormente, las muestras fueron enviadas al laboratorio químico con su respectivo control de calidad (QA/QC), donde se incorporaron muestras duplicadas, estándares y blancos, además, fueron embaladas, etiquetadas y transportadas. Cada envío portaba su respectiva cadena de custodia (ver Figuras 1a y $1 b)$.

\section{ANÁLISIS DE LABORATORIO}

Las muestras obtenidas en los frentes de operación de los dos distritos mineros fueron analizadas en laboratorios certificados con el fin de obtener los resultados requeridos para su análisis. 
El análisis se lo realizó para los siguientes elementos: $\mathrm{Au}, \mathrm{Ag}, \mathrm{Al}, \mathrm{As}, \mathrm{Ba}, \mathrm{Be}, \mathrm{Bi}, \mathrm{Ca}, \mathrm{Cd}, \mathrm{Ce}, \mathrm{Co}, \mathrm{Cr}, \mathrm{Cs}$, $\mathrm{Cu}, \mathrm{Dy}, \mathrm{Er}, \mathrm{Eu}, \mathrm{Fe}, \mathrm{Ga}, \mathrm{Gd}, \mathrm{Hf}, \mathrm{K}, \mathrm{La}, \mathrm{Li}, \mathrm{Lu}, \mathrm{Mg}, \mathrm{Mo}$, $\mathrm{Na}, \mathrm{Nb}, \mathrm{Nd}, \mathrm{Ni}, \mathrm{P}, \mathrm{Pb}, \mathrm{Rb}, \mathrm{S}, \mathrm{Sb}, \mathrm{Sc}, \mathrm{Sn}, \mathrm{Sr}, \mathrm{Ta}, \mathrm{Th}, \mathrm{Ti}$, Tl, U, V, W, Y, Zn, Zr. Se debe considerar que en los resultados estos elementos son similares para los dos casos de estudio.

De los elementos antes mencionados, el Au fue analizado por ensayo al fuego, pero éste no se consideró ya que se presentaba en los dos distritos mineros y es el metal económico para los mineros en ambos yacimientos. En cambio, para el resto de elementos se solicitó un análisis por ICP-OES e ICP-MS, análisis que entregó los resultados en $\mathrm{ppm}, \mathrm{mg} / \mathrm{kg}$; lo que es similar a tener g/ton, que es un término más empleado en minería.

\section{INTERPRETACIÓN}

Con los resultados entregados por los laboratorios de las muestras tomadas en los distritos mineros, se realizó un análisis a 48 elementos, tomando en consideración la concentración de cada elemento para cada distrito minero. Los resultados se evidenciaron por medio del análisis de los datos interpretados, usando estadística descriptiva, y la representación en gráficos de dispersión y asociación de elementos.

Con la interpretación se definió la presencia de concentraciones propias de cada distrito minero, lo que es evidente en la distribución de la nube de puntos de los gráficos de dispersión, tal como lo indica Gehisy (2017).

\section{DISCUSIÓN DE RESULTADOS}

Con los datos obtenidos se realizó un análisis estadístico de los 48 elementos, con el fin de estudiar los resultados de las muestras y poder ir determinando elementos que poseen una gran variación de los resultados en cada uno de ellos.

Aplicando estadística descriptiva a los resultados de las muestras por cada elemento en los dos distritos mineros, se observa variaciones de media (valor promedio), mediana y moda. Con estos valores podemos determinar la diferencia en los promedios de concentración de un elemento en el mineral de cada distrito; por ejemplo, en el análisis de plata, se observa que la media de la concentración obtenida en Zaruma-Portovelo es muy superior a la obtenida en Nambija. Igualmente, los resultados de la desviación estándar son elevados en Zaruma-Portovelo, lo que indica que los resultados de las muestras de plata poseen una mayor dispersión con relación a la media, lo que indica que tienen valores muy variables con relación al promedio (ver Tablas 1 y 2 ).

Otro ejemplo es el erbio (Er), del cual se puede evidenciar un valor promedio alto (media) en Nambija y una mayor dispersión de elementos en los valores representados por la desviación estándar y varianza. Con los datos obtenidos y considerando la amplitud y, por ende, las clases de cada grupo de muestras, se elaboraron los histogramas, los cuales nos indican el número de muestras y su distribución para cada clase; por ejemplo, para el $\mathrm{Pb}$ (ver Figuras 2 y 3 ).

Con los datos entregados por la estadística descriptiva y los histogramas de frecuencias, se definieron las características de concentración que tienen los elementos, mismos que son verificados con los gráficos de dispersión (Figura 4 y Figura 5), con los cuales se deduce que hay elementos con ciertos valores en sus resultados, que se pueden diferenciar en las concentraciones entre los dos depósitos mineros y es así que se describe:

\section{Plata (Ag)}

La plata presenta una mayor concentración en el distrito minero Zaruma-Portovelo, con valores que superan los $8,4 \mathrm{~g} /$ ton (valor máximo de Nambija) en un $74 \%$ de las muestras obtenidas. Aunque hay que considerar que en este distrito también tenemos muestras con resultados de efecto pepita encontrando valores de hasta $200 \mathrm{~g} /$ ton.

\section{Cerio (Ce)}

El cerio se presenta con una mayor concentración en el distrito minero Nambija, con valores que superan los $21,61 \mathrm{~g} / \mathrm{ton}$, lo que corresponde al $67 \%$ de la población de muestras. Razón por la que, el cerio podría ser un elemento indicador del distrito minero Nambija, cuando supera la concentración máxima del otro yacimiento.

\section{Erbio (Er)}

Esta tierra rara presenta una mayor concentración en el distrito Nambija, el valor máximo presente en Zaruma-Portovelo es de 1,53 g/ton, mismo que es superado por el $77 \%$ de las muestras tomadas en Nambija.

\section{Gadolinio (Gd)}

El gadolinio presenta una mayor concentración en el distrito minero Nambija, con valores superiores a los 2,36 g/ton, correspondiente al 79\% de muestras obtenidas en este distrito que son mayores al valor máximo del distrito Zaruma-Portovelo. Además, en Nambija se observó valores de efecto pepita superiores a $10 \mathrm{~g} /$ ton. 

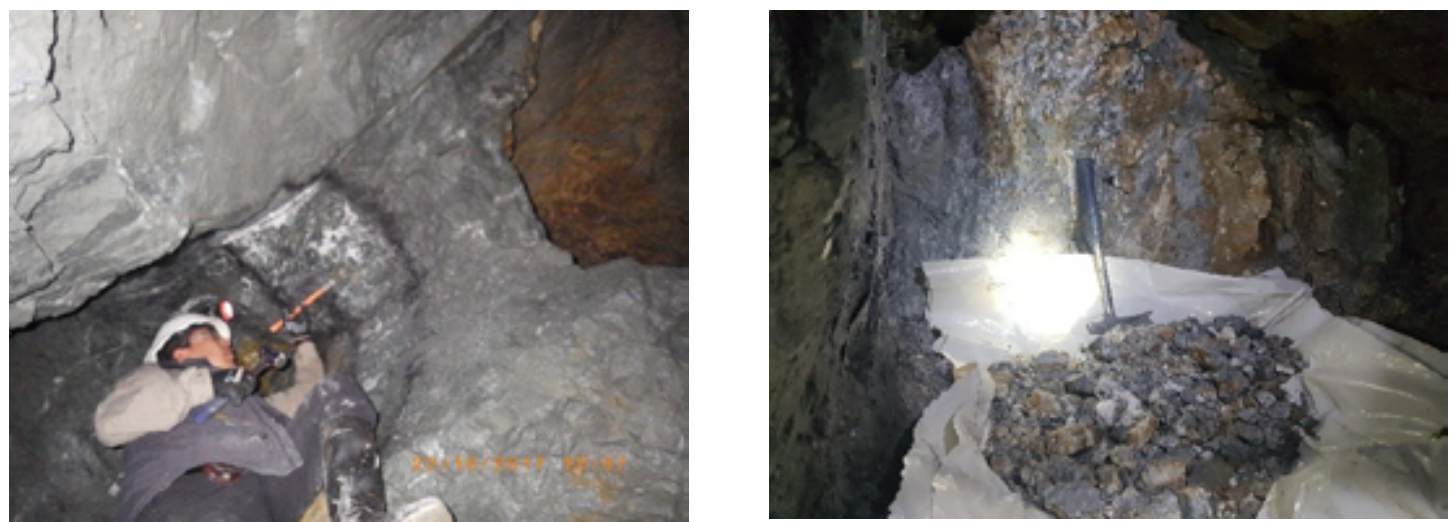

Figura 1. (a) Toma de muestra. (b) Material listo para el cuarteo

Tabla 1. Estadística descriptiva del Ag

\begin{tabular}{lll}
\hline Estadística descriptiva & Ag Nambija & Ag Zaruma \\
\hline Media & 1,47 & 28,15 \\
Error típico & 0,12 & 3,79 \\
Mediana & 1,10 & 15,64 \\
Moda & 0,8 & 101 \\
Desviación estándar & 1,42 & 33,49 \\
Varianza de la muestra & 2,01 & 1121,62 \\
Curtosis & 9,84 & 10,89 \\
Coeficiente de asimetría & 2,94 & 2,77 \\
Rango & 8,30 & 210,28 \\
Mínimo & 0,10 & 0,60 \\
Máximo & 8,40 & 210,88 \\
Suma & 219,80 & 2195,42 \\
Cuenta & 150 & 78 \\
Nivel de confianza (95,0\%) & 0,23 & 7,55 \\
\hline
\end{tabular}

Tabla 2. Estadística descriptiva del Er

\begin{tabular}{lll}
\hline Estadística descriptiva & Ag Nambija & Ag Zaruma \\
\hline Media & 2,36 & 0,54 \\
Error típico & 0,11 & 0,04 \\
Mediana & 2,07 & 0,49 \\
Moda & 2,07 & 0,01 \\
Desviación estándar & 1,29 & 0,37 \\
Varianza de la muestra & 1,66 & 0,14 \\
Curtosis & 13,43 & $-0,37$ \\
Coeficiente de asimetría & 2,64 & 0,53 \\
Rango & 10,74 & 1,52 \\
Mínimo & 0,24 & 0,01 \\
Máximo & 10,98 & 1,53 \\
Suma & 354,46 & 41,94 \\
Cuenta & 150 & 78 \\
Nivel de confianza (95,0\%) & 0,21 & 0,08 \\
\hline
\end{tabular}




\section{Lutecio (Lu)}

En el caso del Lutecio los análisis de las muestras tomadas en Zaruma-Portovelo, están bajo el límite de detección, presentado por el laboratorio y solo 1 muestra de las 78 tomadas presentó un valor de 0,13 g/ton; además, los datos de concentraciones superiores en el distrito minero Nambija corresponden al $90 \%$, razón por la cual, la presencia de este elemento, sea como traza o ultra traza, nos indica que su procedencia es Nambija.

\section{Neodimio (Nd)}

La concentración de este elemento en el distrito minero Nambija, presenta valores sobre el $75 \%$ del valor máximo obtenido en Zaruma-Portovelo de 10,81 g/ton, la mayor concentración se presentaría al sur oriente del país.

\section{Fósforo (P)}

El fósforo tiene una mayor presencia en el distrito minero Nambija, la media que presentan estos valores es casi 6 veces mayor que la presentada en Zaruma-Portovelo. Además, presentan un 55\% de valores superiores al máximo de Zaruma-Portovelo, que es de $600 \mathrm{~g} /$ ton, considerando que éste es un valor de efecto pepita en este distrito y los valores estarían bajo los $400 \mathrm{~g} / \mathrm{ton}$.

\section{Plomo (Pb)}

Así como la plata, de igual forma el plomo presenta una mayor concentración en el distrito minero $\mathrm{Za}$ ruma Portovelo, con valores superiores a $180 \mathrm{~g} / \mathrm{ton}$, que corresponden al 76\% de la población de muestras (ver Figuras 4 y 5). Además, estadísticamente el valor promedio que se presenta en el distrito minero $\mathrm{Za}$ ruma-Portovelo es de $1892 \mathrm{~g} /$ ton, cuyo valor es 125 veces mayor a las concentraciones presentes en Nambija, por tal motivo, concentraciones mayores definen una procedencia.

\section{Uranio (U)}

Este elemento radiactivo se encuentra en los dos depósitos mineros, pero con una mayor concentración en Nambija, con valores sobre $0,45 \mathrm{~g} /$ ton, correspondiente al 89\% de las muestras tomadas en este distrito. Además, en Nambija presenta valores de hasta 9,3 g/ ton de acuerdo a los resultados obtenidos

\section{Itrio (Y)}

Continuando con elementos del grupo de tierras raras, tenemos al itrio, el cual presenta un comporta- miento similar a las anteriores; por ende, es un indicador para determinar que la procedencia es el distrito minero Nambija. El valor máximo de este elemento en el distrito Zaruma es de $11,30 \mathrm{~g} /$ ton, pero las concentraciones mayores a este valor en Nambija corresponden a un $77 \%$ de muestras.

\section{Zirconio (Zr)}

Este elemento presenta mayores valores de concentración en el distrito minero Nambija, con un $61 \%$ de valores sobre la concentración máxima de Zaruma-Portovelo. Razón por la cual podemos definir como un elemento que, considerando cierta concentración o ley, puede ser indicador de que su procedencia es Nambija (ver Figuras 6 a y b). Con el análisis de dispersión se puede observar algunas asociaciones de elementos que se relacionan en cada distrito minero, es así que en Zaruma-Portovelo las concentraciones de $\mathrm{Ag}-\mathrm{Pb}$ son indicadores de su procedencia, lo que se relacionaría con la mineralogía que presenta el depósito y la concentración promedio de estos elementos, tal como lo indica la figura 6.

Para el distrito minero Nambija se puede ver que en los elementos que poseen una alta concentración con relación a los valores promedios, se contemplaría una asociación de tierras raras, tal como lo indica la figura 7 (ver Figura 7).

\section{CONCLUSIONES}

De acuerdo con el análisis realizado, se puede verificar la existencia de diferentes concentraciones de elementos químicos en cada uno de los depósitos minerales

Con el análisis realizado es posible determinar ciertos elementos como indicadores de la procedencia del mineral, es así que en Zaruma-Portovelo, la asociación $\mathrm{Ag}-\mathrm{Pb}$, con valores superiores de plata $(>8,4 \mathrm{~g} /$ ton $)$ y plomo (>181 g/ton), tienen una alta probabilidad de que su origen sea Zaruma-Portovelo (ver Tabla 3).

En cambio, la presencia de tierras raras con concentraciones mayores a Ce ( $>22 \mathrm{~g} / \mathrm{ton}), \operatorname{Er}(>1,6 \mathrm{~g} /$ ton), $\mathrm{Gd}$ (> 2,4 g/ton), $\mathrm{Nd}$ (> 10,81 g/ton), $\mathrm{Y}$ (>11,3 $\mathrm{g} /$ ton), además de la presencia de $\mathrm{Lu}$, nos indica que la probabilidad de que el origen sea Nambija es muy alta, además de otros elementos como el uranio (> $0,91 \mathrm{~g} /$ ton) y zirconio ( $>74 \mathrm{~g} /$ ton $)$ que complementarían esta decisión. 


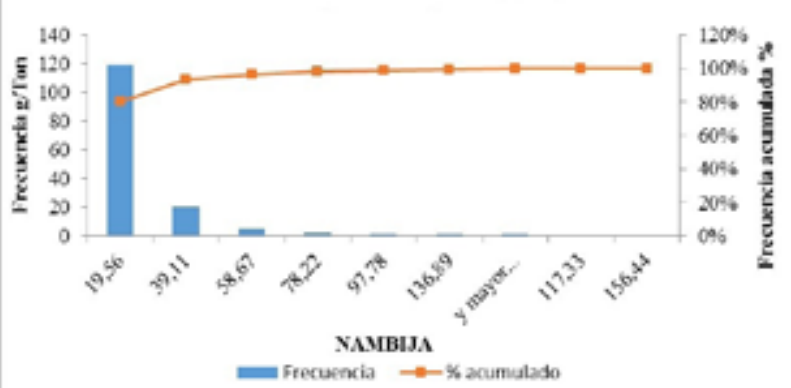

Figura 2. Histograma de frecuencias del $\mathrm{Pb}$, distrito Nambija
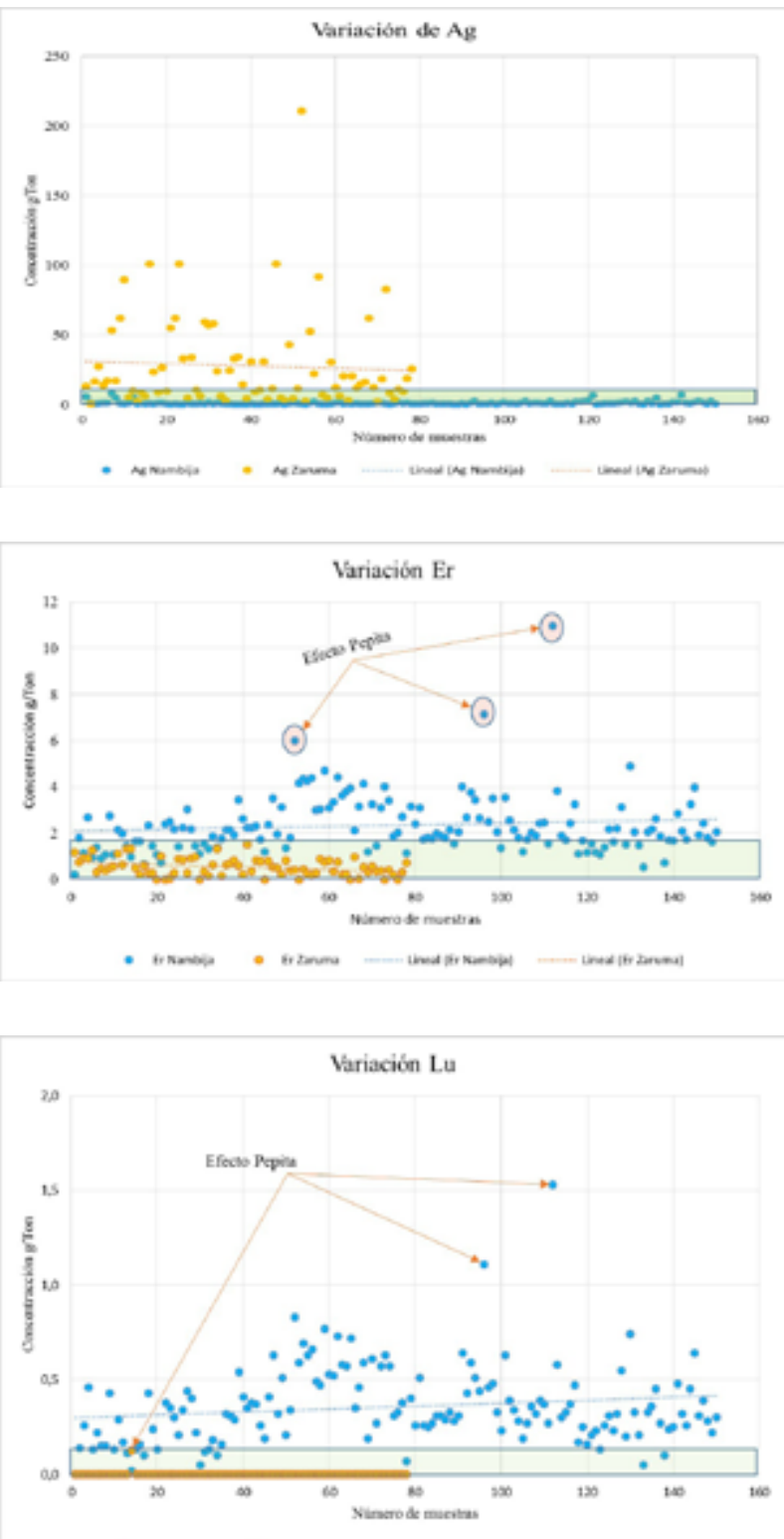

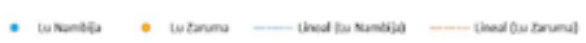

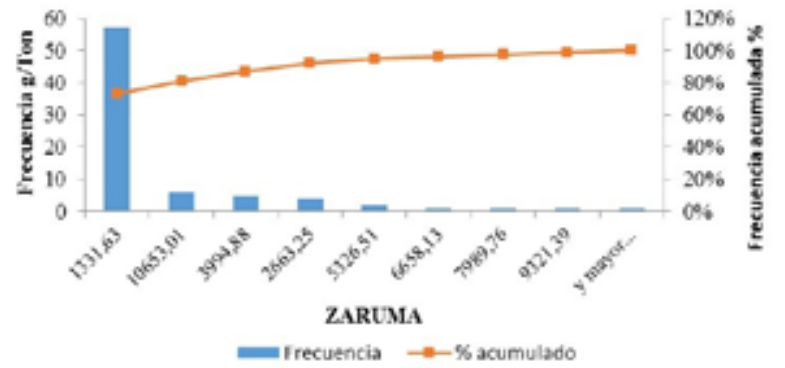

Figura 3. Histograma de frecuencias del $\mathrm{Pb}$, distrito $\mathrm{Za}$ ruma-Portovelo
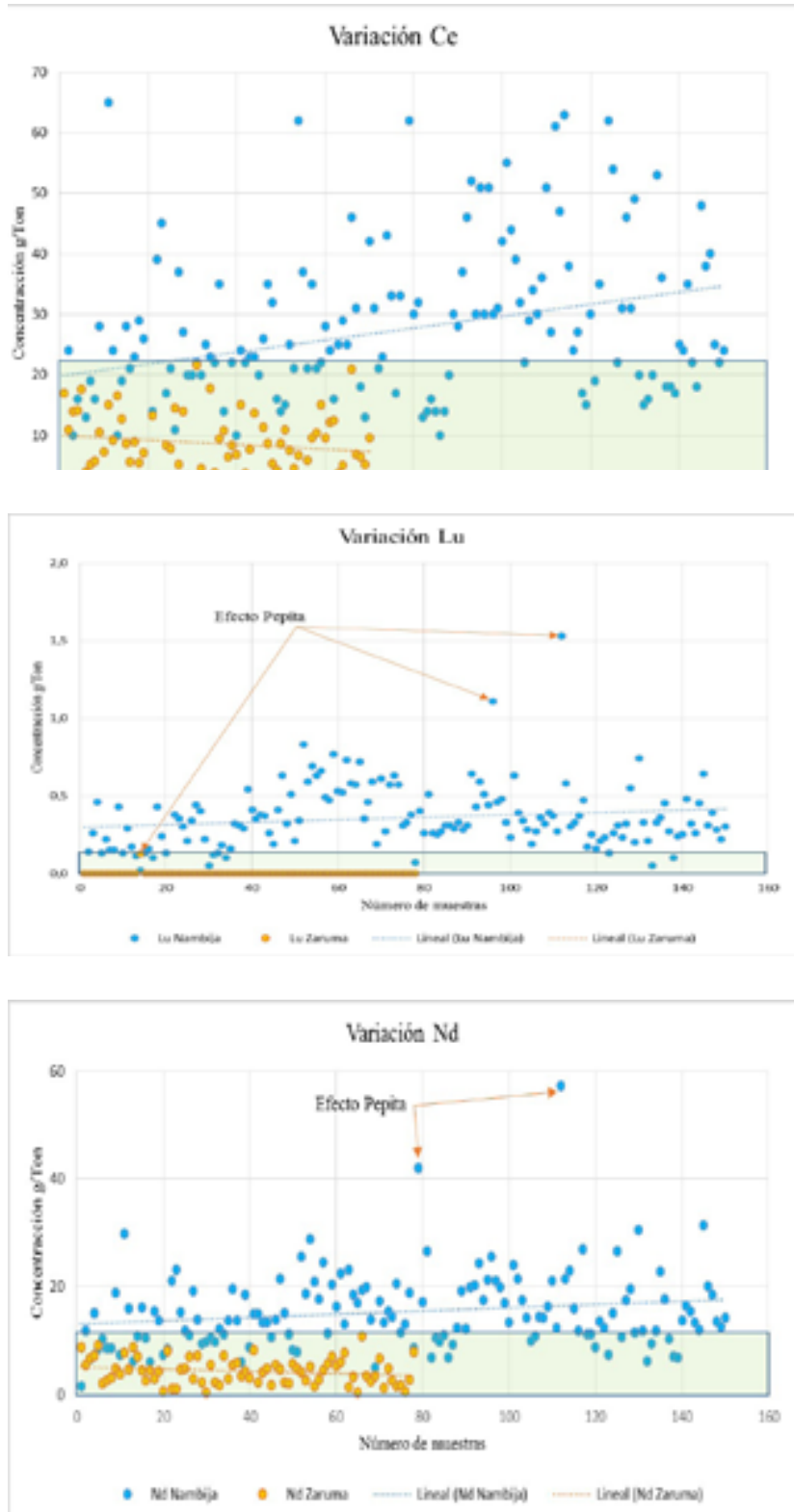

Figura 4. Gráficos de dispersión en relación a la concentración de los elementos: Ag, Ce, Er, Gd, Lu y Nd 

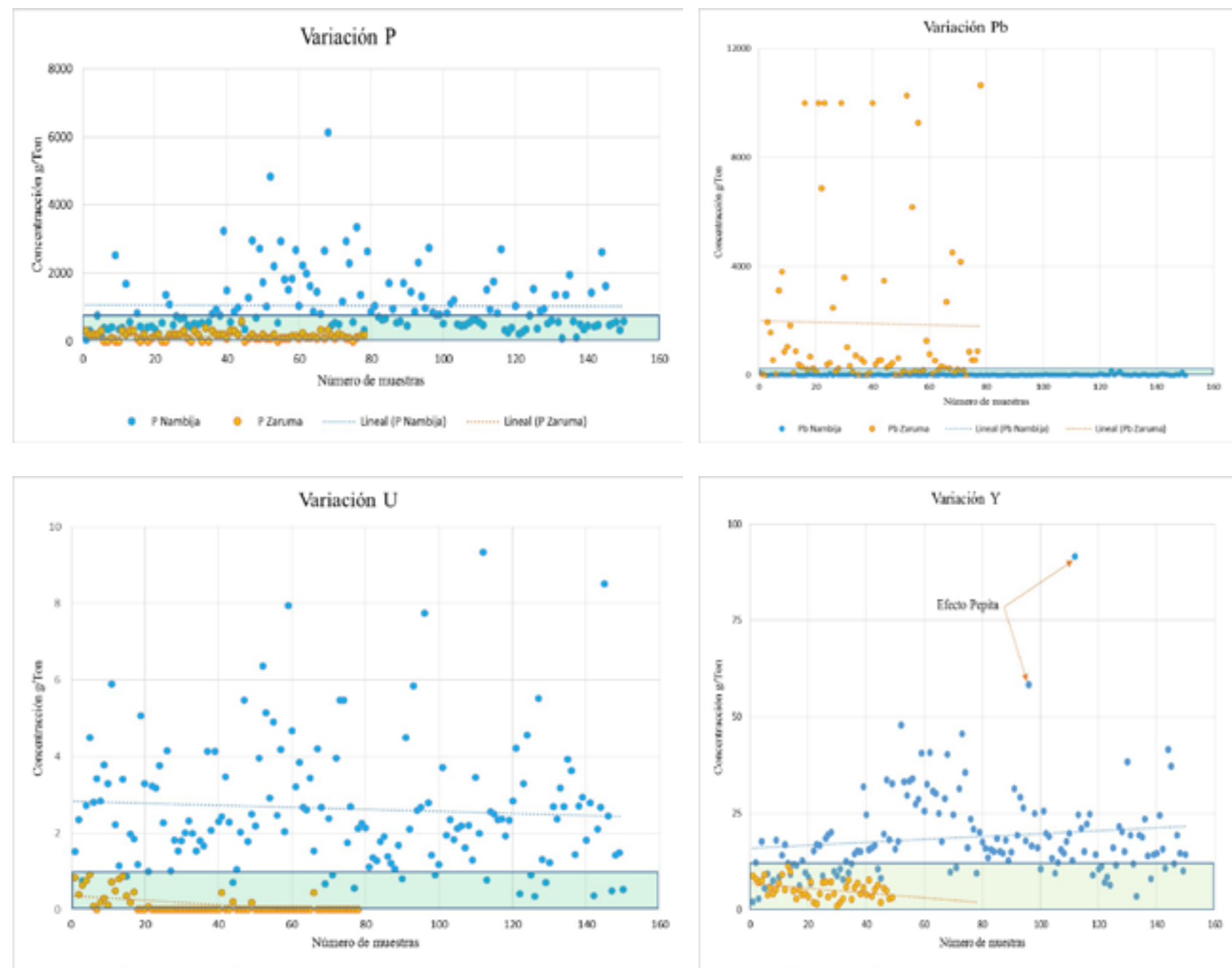

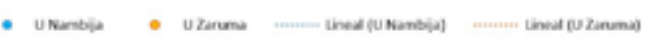

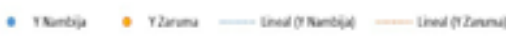

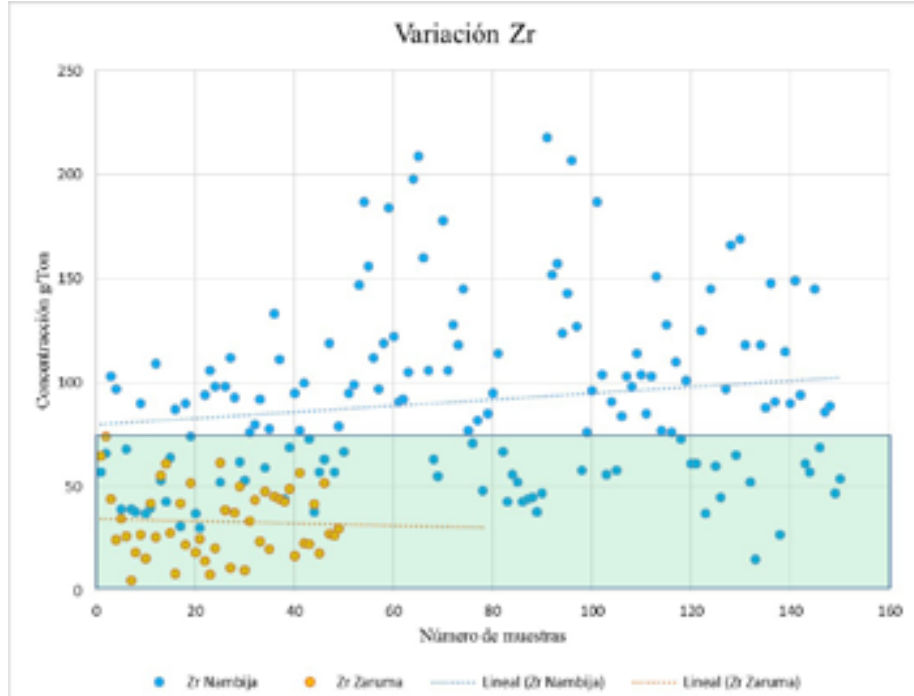

Figura 5. Gráficos de dispersión en relación a la concentración de los elementos: $\mathrm{P}, \mathrm{Pb}, \mathrm{U}, \mathrm{Y}$ y Zr 


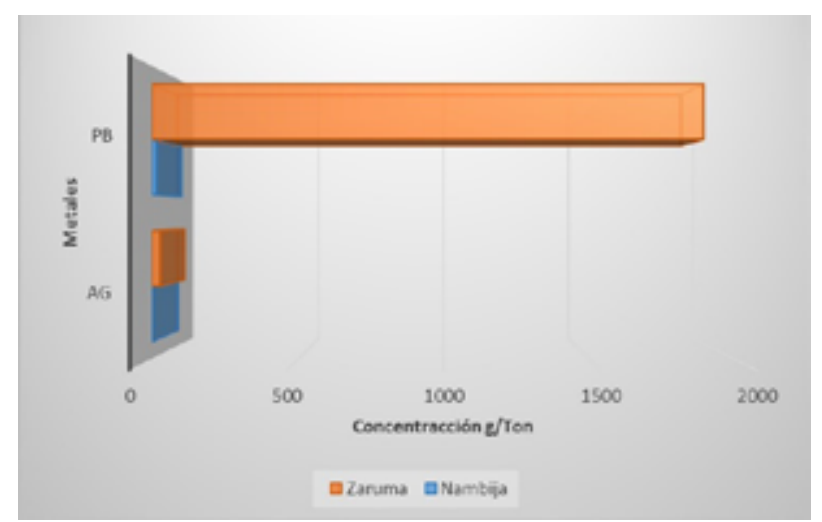

Figura 6a. Asociación de Ag- $\mathrm{Pb}$, con mayor concentración en Zaruma-Portovelo

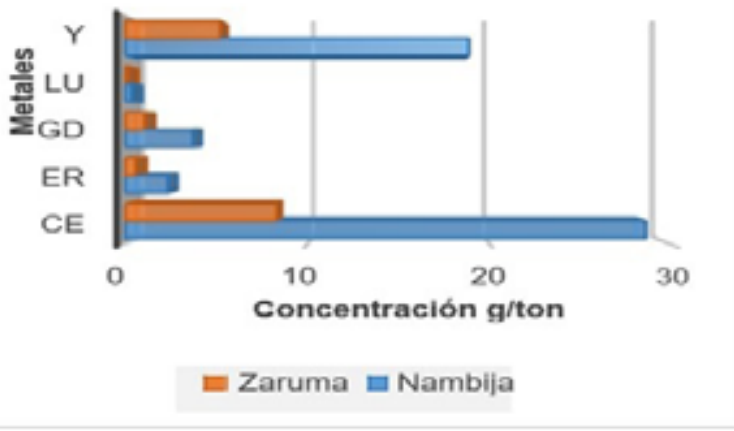

Figura 6b. Asociación de Ce, Er, Gd, Lu e Y, con mayor concentración en Nambija.

Tabla 3. Elementos identificadores por mayor concentración de los distritos Zaruma-Portovelo y Nambija.

\begin{tabular}{|c|c|c|c|}
\hline Elemento & Concentración & Zaruma-Portovelo & Nambija \\
\hline Plata (Ag) & $8,4 \mathrm{~g} /$ ton & $\uparrow$ & \\
\hline Cerio (Ce) & $22 \mathrm{~g} /$ ton & & $\uparrow$ \\
\hline Erbio (Er) & $1,6 \mathrm{~g} / \mathrm{ton}$ & & $\uparrow$ \\
\hline Gadolinio (Gd) & $2,4 \mathrm{~g} / \mathrm{ton}$ & & $\uparrow$ \\
\hline Lutencio $(\mathrm{Lu})$ & $0,13 \mathrm{~g} / \mathrm{ton}$ & & $\uparrow$ \\
\hline Neodimio (Nd) & $10,81 \mathrm{~g} /$ ton & & $\uparrow$ \\
\hline Fósforo (P) & $600 \mathrm{~g} / \mathrm{ton}$ & & $\uparrow$ \\
\hline Uranio (U) & $0,91 \mathrm{~g} / \mathrm{ton}$ & & $\uparrow$ \\
\hline Plomo $(\mathrm{Pb})$ & $181 \mathrm{~g} / \mathrm{ton}$ & $\uparrow$ & \\
\hline Itrio $(\mathrm{Y})$ & $11,30 \mathrm{~g} /$ ton & & $\uparrow$ \\
\hline Zirconio (Zr) & $74 \mathrm{~g} /$ ton & & $\uparrow$ \\
\hline
\end{tabular}

\section{REFERENCIAS}

ACDI-INEMIN. 1987. Informe de prefactibilidad geológico metalogénico de la zona sur del yacimiento aurífero de Nambija. Quito: INEMIN.

Aperin, M. 2013. Introducción al análisis estadístico de datos geológicos. Buenos Aires, Argentina: Red de Editoriales Universitarias Nacionales (REUN).

Bonilla, W. 2009. Metalogenia del distrito minero Zaruma-Portovelo, República del Ecuador. Universidad de Buenos Aires. Portovelo.

British Geological Survey-Corporación de Desarrollo e Investigación Geológico Minero y Metalúrgico. 1997. Mapa geológico de la cordillera Occidental del Ecuador entre 3-4 ${ }^{\circ} \mathrm{S}$, escala 1:200000. Quito.

Delgado, A. E. 2015. Proyecto rehabilitación Nambija. Quito: INIGEMM.
Gallegos, J. 2018. Estos son los metales preciosos que acompañan al oro como los más valiosos y caros del mundo. Recuperado de https://oroinformacion.com/estos-sonlos-metales-preciosos-que-acompanan-al-oro-comolos-mas-valiosos-y-caros-del-mundo/

Ingeoespert. 2017. Tipos de muestreo en minería. Recuperado de: https://ingeoexpert.com/blog/articulo/tipos-muestreo-mineria/

INIGEMM. 2018. Proyecto de trazabilidad del oro desde los frentes de explotación a las plantas de beneficio en $\mathrm{Za}$ ruma-Portovelo. Quito: INIGEMM.

Laboratorio de Técnicas Instrumentales UVa. 2016. Espectroscopía de plasma ICP-OEs. Recuperado de: http:// laboratoriotecnicasinstrumentales.es/analisis-qumicos/ espectroscopa-de-plasma-icp-oes

PRODEMINCA. 2000. Evaluación de distritos mineros del 
Ecuador: potencial minero metálico y guías de exploración (vol. 1). Quito, Ecuador: Ministerio de Energía y Minas.

Salazar, C. y Del Castillo, S. 2018. Fundamentos básicos de estadística (pp. 36-116). Recuperado de: http://www.dspace. uce.edu.ec/bitstream/25000/13720/3/ Fundamentos\%20 B\%C3\%A1sicos\%20de\%20Estad\%C3\%ADstica-Libro.pdf Universidad Complutense de Madrid. (s. f.). Inductively coupled plasma optical emisión spectrometry. Recuperado de: https://www.ucm.es/tecnicasgeologicas/espectro- metria-de-emision-con-fuente-de-ionizacion-icp-oes Universidad de Burgos. 2019. Espectrometría de masas de plasma (ICP-MS). Recuperado de: https://www.ubu.es/ parque-cientifico-tecnologico/servicios-cientifico-tecnicos/espectrometria/espectrometria-de-masas-de-plasma-icp-ms 